\title{
Posttrial injections of strychnine sulfate: Facilitation of consolidation or retrieval?
}

\author{
ARTHUR P. LECCESE and DOUGLAS S. GRANT \\ University of Alberta, Edmonton, Alberta T6G 2E9, Canada
}

\begin{abstract}
The existing literature demonstrating that animals given strychnine sulfate shortly after training display enhanced retention $24 \mathrm{~h}$ later can be interpreted in terms of either consolidation or retrieval processes. A test between these two interpretations failed to support the consolidation interpretation, but did support the retrieval interpretation. It was concluded that (a) a retrieval model provides an adequate interpretation of the facilitation of retention resulting from posttrial injections of strychnine, and (b) an unambiguous demonstration that strychnine enhances processes of consolidation has yet to be offered.
\end{abstract}

A number of studies have demonstrated that injections of strychnine sulfate adminstered shortly after training facilitate performance on a test of retention given 24 h later (e.g., Brennan \& Gordon, 1977; Hudspeth, 1964; Krivanek \& Hunt, 1967). Furthermore, the magnitude of facilitation has been shown to decline as the interval between the end of training and the administration of strychnine increases (e.g., Gordon, 1977; McGaugh \& Krivanek, 1970). The generally accepted interpretation of such findings is that the posttrial administration of strychnine enhances the memory of prior training by facilitating the transition of the memory trace from a labile, temporary state to a static, permanent state, a process referred to as consolidation. Such an interpretation is particularly compelling in light of the accepted notion that the posttrial injection procedure results in the drug not being present at the time of either training or testing (see Dawson \& McGaugh, 1973).

Although the assumptions that the animal is neither trained nor tested under the influence of the drug are critical to the interpretation of posttrial facilitation in terms of consolidation processes, both assumptions are open to question. While it is obvious that animals in such studies are not trained under a drug state, it is conceivable that the physiological consequences of a drug injection given shortly after training may become associated with the memory of that training experience. In other words, even though a

\footnotetext{
This research was conducted by the first author under the direction of the second and was submitted to the University of Alberta in partial fulfillment of the requirements for the MSc degree. The research was supported by thesis funds from the Department of Psychology, University of Alberta, and by Grant A0443 from the National Research Council of Canada to the second author. Arthur P. Leccese is now at the Department of Psychology, University of Louisville, Louisville, Kentucky 40208 . Requests for reprints should be sent to: Douglas S. Grant, Department of Psychology, University of Alberta, Edmonton, Alberta T6G 2E9, Canada.
}

training experience has been completed, the memory of that experience may remain active for some period of time during which it is subject to modification. On this view, an injection that is followed by gross physiological changes would likely be sufficiently salient to be incorporated into the memory of the training experience. As time passes, the training experience memory may become progressively more resistant to modification, resulting in a decreased probability that the physiological consequences of the drug injection will become associated with that memory as the training-injection interval is lengthened. Direct empirical support for the notion that posttrial events can become associated with the memory of a recently completed training experience is provided by Chute and Wright (1973) and Wright and Chute (1973). Following passive avoidance training, animals were injected with either saline or sodium pentobarbital. Prior to retention testing, half of each group received saline and the other half received sodium pentobarbital. Animals tested under the same state as that induced shortly after training performed significantly better than those tested under a state different from that induced shortly after training. These findings suggest strongly that the physiological consequences of a drug administered posttrial can become represented as part of the training memory. As Chute and Wright conclude, "Experimental procedures that give a drug before or after behavioral training may be affected by state dependent learning"' (p. 879, italics added).

The second assumption, that animals are not tested under the influence of the drug, is also open to question. Cooper and Krass (1963) administered a single $1.25-\mathrm{mg} / \mathrm{kg}$ injection of strychnine sulfate either 24 or $72 \mathrm{~h}$ prior to training on a Hebb-Williams maze problem. Relative to noninjected controls, both pretrial injected groups made significantly fewer errors during the $\mathbf{2 0}$ training trials. These data suggest strongly that the drug, or some effect of the drug, was maintained for up to $72 \mathrm{~h}$ following a single injection. 
Moreover, although Alpern and Crabbe (1972) claim to have demonstrated that strychnine administered long after training facilitates subsequent retention, their data may be interpreted more plausibly in terms of strychnine carryover effects. In their study, mice began a regime of 10 daily injections of $.2 \mathrm{mg} / \mathrm{kg}$ of strychnine sulfate $24 \mathrm{~h}$ following training. On a retention test given $24 \mathrm{~h}$ following the final injection, strychnine-treated mice demonstrated better retention than saline-treated mice. Although nontrained controls which received equivalent strychnine injections were included, these animals were tested 48 , rather than 24 , hours after the final injection. Thus, these data may be interpreted as demonstrating a proactive facilitation of retention test performance when the test followed the final injection by $24 \mathrm{~h}$.

Given that the physiological consequences of a drug administered posttrial may become associated with a recently completed training experience and that animals tested $24 \mathrm{~h}$ following injection may still be under the influence of the drug, it is possible to offer an alternative account of the posttrial facilitation studies in terms of Spear's $(1971,1973,1976$, 1978) attribute-retrieval model of animal memory. According to Spear, a memory can be conceptualized as a collection of attributes representing the events noticed by the organism during memory formation. The probability that the animal will manifest retention at the time of testing is directly related to the number and salience of retrieval cues present which correspond to attributes of the to-be-remembered memory. The greater the number and salience of these retrieval cues, the higher is the probability of memory retrieval. On this view, mechanisms of retrieval, rather than consolidation, may be responsible for the better retention test performance demonstrated by animals receiving a strychnine sulfate injection shortly after training. Specifically, the physiological consequences of a drug injected shortly after training become represented as an attribute or attributes of the training experience memory. When tested $24 \mathrm{~h}$ later, some aftereffect of the drug is still present; this acts as a salient and powerful retrieval cue which aids retrieval of the training memory. Animals not injected following training do not have the benefit of this added retrieval cue, and hence perform more poorly. It is also possible that the better retention evinced by injected animals may be due, in part, to a facilitation of sensory, motor, and/or motivational processes during testing as a result of the drug being present in the animal's system (Drill, 1958; Sollmann, 1957; Theologus, Gibby, Gibby, \& Crough, 1968).

A straightforward test between the consolidation and retrieval interpretations would involve administering a posttrial injection of strychnine sulfate and then testing retention at some sufficiently later time (e.g., 1 week) so as to make the possibility of drug carryover to the test unlikely. A consolidation interpretation would predict facilitation despite the long retention interval. In contrast, a retrieval interpretation would predict no facilitation unless animals previously given a posttrial injection were also given a pretest injection. Such a test was performed in the second experiment. Prior to that, however, a preliminary experiment was performed to determine whether rats are capable of discriminating a saline state from a strychnine state. Such an ability would seem essential to the retrieval interpretation.

\section{EXPERIMENT 1}

If the retrieval interpretation of posttrial facilitation is correct in suggesting that the carryover effects of a strychnine injection function as a powerful retrieval cue during testing, it would seem necessary that a rat be able to discriminate a strychnine state from a normal state. The present experiment employed a standard drug-discrimination procedure to determine whether the physiological consequences of a strychnine injection could be discriminated from those of a saline injection.

\section{Method}

Subjects. Ten naive, male Sprague-Dawley rats, approximately 90 days old, were used. The subjects were reduced to and maintained at $85 \%$ of their ad-lib weights.

Apparatus. A standard $\mathrm{T}$-maze with a startbox measuring $10 \times$ $26 \times 13 \mathrm{~cm}$ and a stem portion measuring $10 \times 48 \times 13 \mathrm{~cm}$ was used. Each arm was $10 \times 49 \times 13 \mathrm{~cm}$ and was fitted with a goalbox, measuring $10 \times 12 \times 13 \mathrm{~cm}$, that was attached to the arm at right angles, extending back toward the startbox. This method of attaching the goalboxes was designed to prevent a rat at the choice point from visually determining which goal contained food on any given trial.

Procedure. The subjects received three daily familiarization sessions of $5 \mathrm{~min}$ each. Food pellets were scattered throughout the maze, and the subjects were permitted unimpeded exploration. Following familiarization, drug discrimination training began. Fifteen minutes prior to each training session, the subjects received an intraperitoneal injection of saline or $1.5 \mathrm{mg} / \mathrm{kg}$ of strychnine sulfate. Training sessions were conducted on alternate days (48-h intersession interval) to reduce the likelihood of drug carryover between sessions. The content of the presession injection was randomized to insure that discrimination be based upon drug state. Half of the subjects were required to turn right in the strychnine condition and left in the saline condition; for the remaining subjects, the contingencies were the reverse. Sessions consisted of 10 massed trials with unlimited correction. Correct responses were reinforced with four $45-\mathrm{mg}$ food pellets. Performance on the first trial of each session was used to assess the control exerted by the different states. Training continued until first-trial performance in each session was clearly controlled by the content of the presession injection.

\section{Results and Discussion}

To arrive at our dependent measure, each animal's initial response within a session was classified as correct or incorrect and the number of correct first-trial 


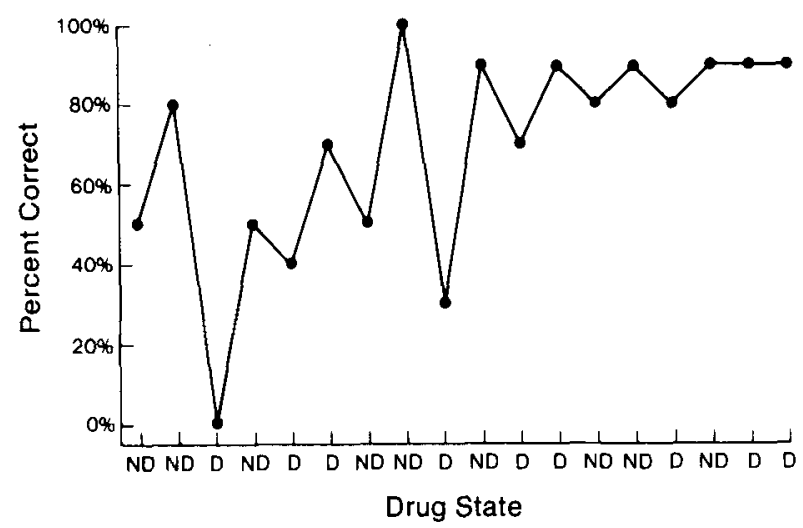

Figure 1. Percentage of correct responses on the first trial of each session as a function of the type of presession injection administered. $\mathrm{ND}=$ saline, $\mathrm{D}=$ strychnine sulfate.

responses was summed across animals and converted to a percentage. In Figure 1, the percentage of animals demonstrating a correct first-trial response as a function of drug state is shown. Since 10 animals were employed, the ordinate may be read as either percent of animals correct or number of animals correct. In the initial sessions, the nature of the presession injection exerted little control over first-trial responding. Control by the nature of the presession injection began to emerge over training. Over the final six sessions, first-trial performance was highly accurate $(86.67 \%)$, indicating that the nature of the presession injection was exerting strong stimulus control over the turning response. Over the final six sessions, four animals made no initial trial errors, four made one error, and two made two errors. These data demonstrate clearly that a rat can discriminate a strychnine state from a normal, saline state.

\section{EXPERIMENT 2}

The present experiment was designed to test between the consolidation and retrieval interpretations of the posttrial facilitative effect of strychnine sulfate. Six groups of rats received training in a Lashley III maze followed by a retention test 7 days later. It was anticipated that the 7-day retention interval would afford sufficient opportunity for the aftereffects of strychnine sulfate injections to completely dissipate. Two groups received strychnine $5 \mathrm{~min}$ after training (D5), two received strychnine $120 \mathrm{~min}$ after training (D120), and two received saline $5 \mathrm{~min}$ after training (N). The 5- and 120-min training-strychnine injection intervals were selected so as to be well within and well outside, respectively, the range of intervals shown to produce memory facilitation (McGaugh \& Krivanek, 1970; McGaugh, Thomson, Westbrook, \& Hudspeth, 1962). Fifteen minutes prior to testing, one group in each condition received an injection of strychnine (D) and the other group received saline $(\mathrm{N})$. The six groups are thus designated D5-D, D5-N, D120-D, D120-N, N-D, and N-N.

The consolidation interpretation predicts facilitation in both DS-D and D5-N, since the strychnine injection is administered during the consolidation period and hence would be expected to enhance that process. On the other hand, D120-D and D120-N should be equivalent to N-D and $N-N$, because the posttrial injection is administered after consolidation has been completed. In contrast, the retrieval interpretation predicts facilitation in D5-D but not in D5-N. In both groups, the physiological consequences of strychnine should become represented as an attribute(s) of the training memory. In D5-D, these consequences would be present during testing as a result of the pretest injection and would serve as a powerful retrieval cue for the training memory. In D5-N, the 7-day retention interval would allow for complete dissipation of the aftereffects of the injection and retrieval of the training memory would be hindered by the change in physiological state from that prevailing during memory formation (i.e., drug) to that prevailing during testing (i.e., normal). Groups D120-D and D120-N receive the strychnine at a point where the training memory is resistant to modification, resulting in the physiological consequences of the strychnine injection failing to be incorporated into the training memory. Therefore, D120-D and D120-N should perform similarly to N-D and N-N. Moreover, the retrieval interpretation anticipates better retention in groups tested in the same state as that prevailing during memory formation (i.e., D120-N and $\mathrm{N}-\mathrm{N}$ ) relative to those tested in a different state from that prevailing during memory formation (i.e., D120-D and N-D). Finally, to the extent that strychnine enhances sensory, motor, and or motivational processes, animals receiving a pretest injection of strychnine would be expected to perform better than those receiving saline pretest. However, in D120-D and N-D, any such facilitation could be masked by a state-dependent retrieval deficit resulting from testing animals in a state different from that prevailing during memory formation. Only in D5-D would such effects not be masked by a state-dependent retrieval deficit; in fact, the beneficial effects of the added retrieval cue involving the physiological consequences of strychnine would be expected to summate with any facilitation of sensory, motor, and/or motivational processes to produce good retention test performance in D5-D.

\section{Method}

Subjects. Sixty-four naive, male Sprague-Dawley rats, approximately 90 days old, were used. The subjects were reduced to and maintained at $85 \%$ of their ad-lib weights.

Apparatus. The animals were pretrained in a straight alley that measured $10 \times 160 \mathrm{~cm}$ and had $23 \mathrm{~cm}-$ high sides. Training and testing during the main experiment were conducted in a 4-unit, 8-cul Lashley III maze (see McGaugh, Thomson, Westbrook, \& 
Hudspeth, 1962, for a complete description). Each unit measured $10 \times 120 \mathrm{~cm}$ and had $13-\mathrm{cm}$-high sides. Choice points were located $30 \mathrm{~cm}$ from the end of culs.

Procedure. The subjects were first pretrained to run a straight alley for four $45-\mathrm{mg}$ food pellets. Five spaced trials were given daily for 7 days. Following pretraining, each rat was assigned randomly to one of six groups: D5-D, D5-N, D120-D, D120-N, N-D, or N-N. Groups Di20-D and N-D each contained 10 subjects, while the remaining groups each had 11 subjects.

All rats were given two rewarded massed training trials in the Lashley IIl maze, followed by one of three treatments: Groups D5-D and DS-N received an intraperitoneal injection of $1.5 \mathrm{mg} / \mathrm{kg}$ of strychnine sulfate $5 \mathrm{~min}$ after training, Groups D120-D and D120-N received a similar strychnine injection 120 min after training, and Groups N-D and N-N received an injection of an equal volume of saline 5 min after training. Pilot work, employing the same subject population and task as the present experiment, demonstrated that a $1.5-\mathrm{mg} / \mathrm{kg}$ injection of strychnine sulfate administered $5 \mathrm{~min}$ after training would facilitate retention on a test given $24 \mathrm{~h}$ later. While this finding is moot with respect to the mechanism responsible for facilitation, it does establish the 1.5$\mathrm{mg} / \mathrm{kg}$ dosage as facilitative in the present population and task

Seven days following training, each rat received five rewarded, massed, retention test trials in the Lashley III maze. Fifteen minutes prior to the test trials, the animals received a $1.5-\mathrm{mg} / \mathrm{kg}$ intraperitoneal injection of strychnine sulfate (D5-D, D120-D, and $\mathrm{N}-\mathrm{D}$ ) or a similar injection of saline (DS-N, DI20-N, and N-N). Following the procedure employed by Greenough and McGaugh (1965) and McGaugh et al. (1962), retracing was permitted but only the initial response at each choice point was scored as correct or incorrect. Errors were scored when a rat's head crossed a white line located $5 \mathrm{~cm}$ into a blind alley. A second observer (Linda Leccese), who was blind with respect to the assignment of animals to treatments, simultaneously recorded errors during all training and testing sessions. The experimenter (Art Leccese) and second observer agreed on about $95 \%$ of choice response classifications; all instances of disagreement were attributable to the second observer's being more conservative in the classification of a response as an error. Most importantly, interobserver reliability was not affected by the treatment to which an animal was assigned.

\section{Results}

The mean number of errors on each of the two training trials and on each of the five retention test trials is shown in Table 1. An analysis of variance revealed that the groups did not differ during the training period $[F(5,58)=1.47, p>.05]$, indicating that differences in retention were not confounded by differences in degree of learning. Retention performance on the first test trial warrants close examination, since performance on subsequent test trial's could be influenced by learning occurring during the test trials. This could be particularly problematic if one assumes that animals given strychnine pretest might learn more quickly than those given saline pretest. Examination of first trial test performance reveals that Group D5-D demonstrated considerable facilitation of retention whereas Group D5-N did not. Furthermore, groups predicted to be in the same state shortly after training and at testing (D5-D, D120-N, and N-N), $\bar{X}=3.42$, made fewer errors than those predicted to be in different states (D5-N, D120-D, and $N-D), \bar{X}=4.68$. The superior performance of same-state groups was consistent within each level of posttraining treatment. These findings were supported by an analysis of variance (Posttraining Treatment by Pretest Treatment) performed on first-trial retention scores, which revealed a significant effect of posttraining treatment $[F(2,58)=5.28, p<.01]$ and a significant Posttraining by Pretest Treatment interaction $[F(2,58)=16.15, p<.001]$. Subsequent Newman-Keuls tests revealed Group D5-D to be superior to all other groups ( $p<.01$ in all cases), while no other differences reached significance at the .05 level.

Examination of the error data for all five test trials revealed a pattern of findings similar to those found using only the first retention test trial. That is, Group D5-D made significantly fewer errors than any other group $(p<.01)$ while no further comparisons were significant and groups predicted to be tested under the same state as that prevalent shortly after training performed significantly better than groups experiencing a change in state, $\bar{X}=2.41$ and $3.85[F(2,230)=188.10, p<.001]$. Examination of the error data on all five test trials also reveals a tendency for all groups to commit fewer errors across trials $[\mathrm{F}(4,40)=66.03, \mathrm{p}<.01]$, although the trials factor did not interact with either the posttraining or pretest treatment.

\section{Discussion}

The findings of the present experiment provide no support for an interpretation of the facilitative effect of posttrial injection of strychnine sulfate in terms of the enhancement of consolidation processes. Animals receiving strychnine shortly after training do not demonstrate enhanced retention under conditions which eliminate the possibility that the drug

Table 1

Mean Number of Errors on Each of the Two Training Trials and on Each of the Five Test Trials as a Function of Condition in Experiment 2

\begin{tabular}{|c|c|c|c|c|c|c|c|c|}
\hline \multirow[b]{2}{*}{ Condition } & \multicolumn{2}{|c|}{ Training Trial } & \multicolumn{6}{|c|}{ Test Trial } \\
\hline & 1 & 2 & 1 & 2 & 3 & 4 & 5 & Mean \\
\hline DS-D & 5.27 & 5.09 & 2.45 & 1.91 & 1.45 & .82 & .63 & 1.45 \\
\hline D5-N & 5.45 & 4.45 & 4.45 & 4.18 & 3.91 & 3.09 & 3.00 & 3.74 \\
\hline D1 20-D & 5.30 & 4.30 & 4.70 & 4.70 & 4.30 & 3.70 & 3.30 & 4.14 \\
\hline D1 20-N & 5.45 & 4.00 & 3.81 & 3.54 & 2.91 & 2.54 & 1.73 & 2.91 \\
\hline $\mathrm{N}-\mathrm{D}$ & 4.80 & 3.80 & 4.80 & 3.70 & 3.40 & 3.40 & 3.10 & 3.68 \\
\hline $\mathrm{N}-\mathrm{N}$ & 5.09 & 4.55 & 4.00 & 3.45 & 2.73 & 2.18 & 2.00 & 2.87 \\
\hline
\end{tabular}


may be present in the animal's system at testing. On the other hand, the findings are consistent with a retrieval interpretation. Specifically, a posttrial injection of strychnine results in enhanced retention test performance only if the physiological consequences of the drug are present at testing. Moreover, animals tested under the same state as that prevailing during memory formation perform better than those experiencing a change in state.

It should be noted that Greenough and McGaugh (1965), following a procedure highly similar to that employed here, claim to have demonstrated enhanced retention in a group comparable to our D5-N. Closer examination of their data, however, provides cause to doubt the validity of their conclusion. In their study, rats received an intraperitoneal injection of $1.0 \mathrm{mg} / \mathrm{kg}$ of strychnine sulfate shortly following the completion of two massed trials in a Lashley III maze. One week later, the animals received five retention test trials. Neither males nor females differed from controls when all five test trials were considered. However, an examination of only the first three test trials reveals that females differed from controls but that males again showed no difference. The post hoc nature of Greenough and McGaugh's analysis, the fact that males showed no facilitation, and our own failure to find facilitation under such conditions combine to suggest that Greenough and McGaugh may have committed a Type I error. A more convincing demonstration would be required to contradict the present conclusion that a posttrial injection of strychnine sulfate will facilitate retention only if the physiological consequences of the drug are present at testing.

\section{GENERAL DISCUSSION}

We have argued that the literature on the facilitative effect of posttrial injection of strychnine sulfate is interpretable in terms of mechanisms of either consolidation or retrieval. The second experiment was designed to differentiate empirically between these two alternatives. The prediction derived from the consolidation interpretation was contradicted by the data, whereas all findings were consistent with the retrieval interpretation. Unfortunately, the experiment did not permit the assessment of the possible role played by an enhancement of sensory, motor, and/or motivational processes during testing which might result from the presence of strychnine in the animal's system.

In addition to both the findings of the present study and the fact that prior studies are amenable to such an interpretation, at least two further reasons may be cited to justify considering seriously the possibility that mechanisms of retrieval, rather than consolidation, mediate the facilitative effect of posttrial injection of strychnine. First, while many mem- ory theorists may endorse the essential notion of consolidation, that a memory undergoes a gradual transition from a labile, temporary state to a static, permanent state, the events transpiring during this transition remain unspecified. Even more mysterious is the mechanism whereby strychnine sulfate might facilitate the events transpiring during the transition. On the other hand, the retrieval interpretation removes much of the mystery concerning the mechanism whereby strychnine enhances retention and allows one to view the physiological consequences of strychnine as a very salient and powerful contextual cue, not qualitatively unlike other contextual cues such as smells, tastes, sounds, or sights.

A second justification for entertaining the retrieval interpretation comes from the work of Gordon (1977) and Gordon and Spear (1973). Following initial passive avoidance training, rats spent 3 days in their home cages. After this 72 -h period, rats received a treatment, a 60-sec exposure to the CS in the training apparatus in the absence of shock, designed to reactivate the passive avoidance memory. Animals receiving a $1.5-\mathrm{mg} / \mathrm{kg}$ injection of strychnine immediately after reactivation demonstrated enhanced retention, relative to those given saline following reactivation, on a test given $24 \mathrm{~h}$ later. Gordon (1977) has shown further that as the reactivation-injection interval is lengthened, the magnitude of the enhancement declines. These findings are problematic for a consolidation interpretation, as one would have to assume that a previously consolidated memory of a welllearned response undergoes reconsolidation upon reactivation, an assumption seemingly inconsistent with common conceptions of the consolidation process. On the other hand, it is reasonable to assume that a reactivated memory would be subject to modification and that, therefore, the physiological consequences of the strychnine injection would become incorporated into the training memory. This process, coupled with the carryover of strychnine effects to the test given $24 \mathrm{~h}$ postinjection, would account for the enhanced retention of passive avoidance.

It may be noted that the present contextual cuebased retrieval interpretation of the facilitative effect of posttrial injection of strychnine sulfate makes contact with recent interpretations of retrograde amnesia which have also emphasized the process of retrieval rather than storage. Such models have been proposed to account for the amnesic properties of the posttrial administration of electroconvulsive shock (ECS; Miller \& Marlin, 1977; Miller \& Springer, 1973; Thompson \& Grossman, 1972; Thompson \& Neely, 1970) and the posttrial induction of hypothermia (Hinderliter, Webster, \& Riccio, 1975; Mactutus \& Riccio, 1978; Riccio, Mactutus, Hinderliter, \& McCutcheon, 1979).

More specifically, our model most closely resembles that of Riccio and his associates (see especially 
Hinderliter et al., 1975), who have proposed a contextual cue interpretation of retrograde amnesia. According to this account, memories remain active and susceptible to modification for some period following termination of the learning experience. Therefore, the stimuli associated with ECS or hypothermia become represented as attributes of the training experience memory when these states are induced shortly after the completion of training. To the extent that animals are tested for retention in the absence of cues associated with the amnesic agent, the change in context will result in a retrieval deficit and amnesia will be demonstrated.

The contextual cue interpretation of retrograde amnesia is similar to, but not identical to, models emphasizing processes of state dependency. While maintaining the virtue of accounting for the fact that pretest reexposure to the amnesic agent attenuates the retention deficit (e.g., Riccio, Mactutus, Hinderliter, \& McCutcheon, 1979; Thompson \& Neely, 1970), the contextual cue model avoids many of the pitfalls encountered by the state dependency model. For example, Thompson and Grossman (1972), using ECS, and Hinderliter et al. (1975), using hypothermia, have found that pretest reexposure to the amnesic agent markedly attenuates the retention deficit while a pretest administration of the amnesic agent impairs retention only slightly for animals not also experiencing the agent posttraining. Hinderliter et al. have argued that such an asymmetry is difficult to reconcile with a state dependency account, but is not problematic for a contextual cue account. According to the contextual interpretation, contextual cues associated with the amnesic agent are essentially irrelevant for control animals since such cues were not present during memory formation. Therefore, the introduction of such cues at the time of testing would be expected to produce only a slight decrement in retrieval effectiveness.

As a second example of the greater power of the contextual cue model as opposed to the state dependency model, consider the findings of Miller, Malinowski, Puk, and Springer (1972). Using a stepthrough passive avoidance situation, Miller et al. found that pretest exposure to footshock attenuated amnesia to a greater extent than did pretest exposure to ECS and that the effect of reexposure was relatively durable in that amnesia was attenuated even when the reexposure occurred 4 days prior to retention testing. While both of these findings are obviously inconsistent with a state dependency model of retrograde amnesia, they do not contradict the contextual cue interpretation. This is because the latter interpretation views the physiological consequences of amnesic agents as contextual stimuli, not qualitatively unlike other contextual stimuli. Therefore, it is not surprising that other salient aspects of context (such as the presence of footshock) might also attenuate amnesia when reintroduced prior to testing. Moreover, the temporal durability of the reexposure effect is not inconsistent with the contextual cue model, since the model is essentially moot with respect to the anticipated duration of the effectiveness of reexposure treatments.

The analyses of the memorial effects of posttraining events offered by Riccio and his associates and ourselves share several features in common. Each model proposes that the training experience memory remains active for some finite period after the termination of the training experience. During this period, the memory is susceptible to modification and will become embedded in a contextual state induced by ECS, hypothermia, or strychnine sulfate administered shortly after training. Finally, each model proposes that retention performance is influenced strongly by contextual stimuli such that the greater the number of salient contextual stimuli present at testing which correspond to attributes of the stored memory, the better will be retention performance.

On the other hand, there is one fundamental difference between our model and that of Riccio. That is, our model accounts for the facilitation of retention produced by strychnine, while Riccio's model accounts for the inhibition of retention produced by ECS and hypothermia. We have argued that the physiological consequences of a strychnine sulfate injection administered shortly after training became represented as an attribute(s) of the training memory. Because the physiological consequences are highly salient and uniquely associated with the training memory, the presence of such consequences at the time of testing represents the presence of extremely powerful retrieval cues which function to increase retention beyond the level demonstrated by control subjects not experiencing strychnine effects both shortly after training and at testing. It appears, then, that our model would predict that the posttraining and pretest administration of ECS or hypothermia should result in the facilitation of retention, rather than simply bringing performance up to control levels. It is possible to suggest, however, at least three reasons why a facilitation of retention has not yet been demonstrated when either ECS or hypothermia has been induced both shortly after training and before testing. First, one could argue that the physiological consequences of ECS and hypothermia are not as salient as those associated with strychnine. This explanation seems somewhat unlikely in that both of these agents produce massive physiological insult usually leading to unconsciousness. A second, more plausible, account involves the notion that ECS and hypothermia may have additional effects on retention. That is, in addition to serving as powerful contextual stimuli, these agents may also tend to produce some disruption of storage or coding processes (Lewis, 1976). If such were the case, these antagonis- 
tic processes (storage or coding disruption and retrieval facilitation) might interact in animals receiving amnesic agents both posttraining and pretest, to produce levels of retention comparable to those of untreated controls. Finally, it may be suggested that methodological difficulties have precluded the demonstration of enhanced retention in animals receiving amnesic agents both posttraining and pretest. For example, since ECS and hypothermia induce immobility which persists for some time following administration, subjects given a pretest treatment are not tested until they appear to be recovered, a process taking between $15 \mathrm{~min}$ and $2 \mathrm{~h}$. It may be that animals so treated have not fully recovered complete cognitive and/or motor functioning at the time of testing. It may also be that by the time the animals recover sufficiently to be tested, the contextual state has weakened beyond the point where facilitation of retention above control levels would be demonstrated.

Whatever the merits of the mechanisms suggested above to account for the discrepancies between the action of ECS and hypothermia on the one hand and strychnine on the other, it seems not unreasonable to suggest that amnesic agents (ECS and hypothermia) may produce effects through many, if not all, of the same mechanisms as those through which strychnine produces retention enhancement. Indeed, the exciting possibility of a theoretical rapprochement of the mechanisms of amnesia and retention enhancement warrants additional empirical work.

By way of a caveat, it should be noted that we are not suggesting that it is unreasonable to anticipate that certain agents may facilitate or hinder processes of consolidation. We do wish to suggest, however, that a convincing demonstration of such an effect in the case of strychnine has yet to be offered. Moreover, the present work suggests that researchers undertaking studies of the effects of various agents on memory should consider carefully how their treatment might affect the retrieval process before concluding that a treatment necessarily influences storage processes.

\section{REFERENCES}

Alpern, H. P., \& Crabbe, J. C. Facilitation of the long-term store of memory with strychnine. Science, 1972, 177, 722-724.

Brennan, M. J., \& Gordon, W. C. Selective facilitation of memory attributes by strychnine. Pharmacology, Biochemistry \& Behavior, 1977, 7, 451-457.

Chute, D. L., \& Wright, D. C. Retrograde state dependent learning. Science, 1973, 180, 878-880.

Cooper, R. M., \& Krass, M. Strychnine: Duration of the effects on maze-learning. Psychopharmacologia, 1963, 4, 472-475.

Dawson, R. G., \& McGaugh, J. L. Drug facilitation of learning and memory. In J. Anthony Deutsch (Ed.), The physiological basis of memory. New York: Academic Press, 1973.

Drill, V. A. Pharmacology in medicine (2nd ed.). New York: McGraw-Hill, 1958.

Gordon, W. C. Susceptibility of a reactivated memory to the effects of strychnine: A time-dependent phenomenon. Physiology \& Behavior, 1977, 18, 95-99.
Gordon, W. C., \& Spear, N. E. The effects of strychnine on recently acquired and reactivated passive avoidance memories. Physiology \& Behovior, 1973, 10, 1071-1075.

Greenovgh, W. T., \& McGaugh, J. L. The effect of strychnine sulfate on learning as a function of time of administration. Psychopharmacologia, 1965, 8, 290-294.

Hinderliter, C. F., Webster, T., \& Riccio, D. C. Amnesia induced by hypothermia as a function of treatment-test interval and recooling in rats. Animal Learning \& Behavior, 1975, 3, 257-263.

Hudspeth, W. J. Strychnine: Its facilitating effect on the solution of a simple oddity problem by the rat. Science, 1964, 145, 1331-1333.

KrivaneK, J. A., \& Hunt, E. The effects of posttrial injections of pentylenetetrazole, strychnine and mephenesin on discrimination learning. Psychopharmacologia, 1967, 10, 189-195.

LEWIS, D. J. A cognitive approach to experimental amnesia. American Journal of Psychology, 1976, 89, 51-80.

Mactutus, C. F., \& Riccio, D. C. Hypothermia-induced retrograde amnesia: Role of body temperature in memory retrieval. Physiological Psychology, 1978, 6, 18-22.

McGaugh, J. L., \& Krivanek, J. A. Strychnine effects on discrimination learning in mice: Effect of dose and time of administration. Physiology \& Behavior, 1970, 5, 1437-1442.

McGaugh, J. L., Thomson, C. W., Westbrook, W. H., \& HUDSPETH, W. J. A further study of learning facilitation with strychnine sulfate. Psychopharmacologia, 1962, 3, 352.360.

Miller, R. R., Malinowski, B., Puk, G., \& Springer, A. D. State-dependent models of ECS-induced amnesia in rats. Journal of Comparative and Physiological Psychology, 1972, 81, $533-540$.

Miller, R. R., \& Marlin, N. A. Amnesia following electroconvulsive shock. In J. F. Kihlstrom \& F. J. Evans (Eds.), Disorders of memory function. Hillsdale, N.J: Erlbaum, 1977.

Miller, R. R., \& Springer, A. D. Amnesia, consolidation and retrieval. Psychological Review, 1973, 80, 69-79.

Riccio, D. C., Mactutus, C. F., Hinderliter, C. F., \& McCutcheon. K. Severity of amnesia and the effectiveness of reactivation treatment: Evidence for a retrieval hypothesis. Physiological Psychology, 1979, 7, 59-63.

Sollmann, T. A manual of pharmacology and its applications to therapeutics and toxicology (8th ed.). Philadelphia: Saunders, 1957.

Spear, N. E. Forgetting as retrieval failure. In W. K. Honig \& P. H. R. James (Eds.), Animal memory. New York: Academic Press, 1971.

Spear, N. E. Retrieval of memory in animals. Psychological Review, 1973, 80, 163-194.

Spear, N. E. Retrieval of memories: A psychobiological approach. In W. X. Estes (Ed.), Handbook of learning and cognitive processes: Attention and memory (Vol, 4). Hillsdale, N.J: Erlbaum, 1976.

SPEAR, N. E. The processing of memories: Forgetting and retention. Hillsdale, N.J: Erlbaum, 1978.

Theologus, G. C., Gibay, R. G., Gibby, R. G., \& Crough, D. G. Enhancement of exploratory behavior by strychnine sulfate. Psychological Reports, 1968, 23, 876-878.

Thompson, C. I., \& Grossman, L. B. Loss and recovery of long-term memories after ECS in rats: Evidence for statedependent recall. Journal of Comparative and Physiological Psychology, 1972, 78, 248-254.

Thompson, C. I., \& Neely, J. E. Dissociated learning in rats produced by electroconvulsive shock. Physiology \& Behavior, $1970,5,783-786$.

Wright, D. C., \& Chute, D. L. State dependent learning produced by posttrial intrathoracic administration of sodium pentobarbital. Psychopharmacologia, 1973, 31, $91-94$.

(Received for publication April 16, 1979; revision accepted September 17, 1979.) 